\title{
PSYCHE.
}

\section{A GENERAL SURVEY OF THE MODES OF DEVELOPMENT IN INSECTS AND THEIR MEANING.*}

The mode of development in all of the first series of orders from I-IX [see Psyche v. 6, p. 13] is as a rule direct, and this necessarily unites the Thysanuriform larva, when it is present, more or less closely with the adult stages, and the adults are apt to show traces of this connection in the retention of certain primitive characteristics. The absence of a waist or deep constriction between the thorax and the abdomen is due to the fact that the junction with the metathorax remains in most adults as it is in the larva and in Thysanura. The mouth parts also are for biting, except in the highly specialized Hemiptera, in which, although the suctorial characteristics of these parts are developed early, the larvae, with this exception, have what may be called a Thysanuriform stage. The highly specialized adults of groups having indirect development (Coccidae) are not exceptions to this rule, and retain to a recognizable degree the primitive form of the larvae.

The second series of orders from $X$ XVI have, as a rule, more complicated modes of development, introducing various intermediate and often extraordinary stages, such as grubs, caterpillars,

* From Guides for Science Teaching, No. VIII. By Alpheus Hyatt and J. M. A rms. etc. Following Brauer and some other entomologists, we have regarded these as more or less degraded modifications of the primitive Thysanuriform larva, but have spoken of them collectively as the secondary larval stages. They appear subsequently to the Thysanuriform stage, when that is present, or between the ovarian and pupal stages when that is absent. The pupal stage is similar to that of the first series of orders in all respects except that, as a rule, it is incapable of motion, or is what is called quiescent, and is usually more or less protected. The complicated development of individuals in the second series of orders has led several authors to designate the first series of orders as Ametabola, and the second series as Metabola.

The use of the term "ametabola," as applied to the orders from I to IX, involves an exaggeration, since it implies that they have no metamorphoses; whereas, as pointed out by Comstock and others, the Coccidae have a "complete" series of metamorphoses, or indirect development, even including a quiescent pupal stage in the development of the only winged form, the male. The quiescence of the pupal stage loses much significance in view of 
this exception, and also when it is noted that an extra quiescent larval stage may occur in the second series of orders, as in some beetles, whose extraordinary habits render two quiescent stages essential in their development.

It is a remarkable fact that, as a rule, the larvae of the second or specialized series of orders have the habit of feeding voraciously. In this way the larvae store up fats and food matters in their own bodies in preparation for the quiescent and helpless pupal stage, during which they live upon these accumulations, they being taken up by the cells of the tissues and used in building up the organs and parts of the adult. The pupal stage is passed, as a rule, in more or less sheltered situations, and it is either enclosed in a special covering, a cocoon, woven by the animal, or else protected by one acquired through the moulting and hardening of its own cuticle. The difference between this last and the ordinary process of moulting consists in the retention of the moulted skin, the animal shrinking within it for shelter as its fatty parts are consumed, instead of casting it off altogether.

Lubbock, in his Origin and metamorphoses of insects, has shown that the inactivity of the pupa in the second series of orders is not a novel condition, but a mere prolongation of the shorter periods of inactivity which necessarily accompany every change of skin or moult. These facts and the obvious want of any common structural differences in the quiescent pupae, as compared with the similar stages of ac- tive pupae, show that quiescence must be reckoned as a habit of resting from active exertion during a more or less prolonged period of their growth which has been acquired by the more specialized forms of insects, not only generally among the members of the second series of orders, but also by many among the first series. The degraded larvae of individuals in these specialized forms are as a rule farther removed structurally from their own adults, than in forms having a direct mode of development, and the changes to be gone through before reaching the adult stage are greater and more numerous. The habits of the animal during the pupal stage have consequently changed in proportion to these requirements from the active to the quiescent condition.

There are other series of facts equally important and significant. While the Thysanuriform stage is present more or less in Coleoptera and Neuroptera, which have the indirect mode of development, it is absent in the orders from XII to XVI inclusive, having been replaced by the secondary larval stages in accordance with the law of acceleration in development.

The tendency of the more specialized forms in the orders I to IX to accelerate the development of the earliest stages is shown in various ways. In the grass. hoppers,* Mantidae, etc., the inheritance of the adult peculiarities of the type affects the young at such early

\footnotetext{
* Packard's illustrations on p. 60 of his Entomology for beginners give an excellent series of one species, Caloptenus femur- rubrum
} 
stages that, as has been described above, the primitive larval 'Thysanuriform stage is skipped or omitted from the development.

In Coleoptera and in the highly specialized orders of insects (XI to XVI) a novel and disturbing influence appears, due to the extraordinary importance of the functions of larval life. This period in the larger number of groups in other classes of animals is much less variable than the adult stage, and it is really very often a mere vehicle for the record and transmission of hereditary characters. In some of the orders of insects, however, it is as efficient for the manifestation of new modifications and adaptive characters as the adult, and often perhaps more variable. This is an exceptional rather than the usual aspect of the larval stages, and makes the study of insects remarkably difficult and interesting.

Sometimes in the orders I to IX (Coccidae, Cicada), as well as more generally in $\mathrm{X}$ to XVI, the larvae carry the line of development and modification a long way outside of what can be termed the normal or direct course, but these deviations lead, as a rule, back again through similar pupae to the same goal in the imago, a typical adult insect. Epicauta, the blister-beetle, is a good example. Fig. 9S shows the active Thysanuriform larva, and Figs. I02, I06, I07, the grub-like larva which passes through two stages before becoming the true pupa that transforms into the imago. These complications were probably due originally in each type to the plastic nature of the organism, which enabled it to fit itself to different conditions and surroundings during its passage through the younger stages of growth. The history of parasites, whose loss of parts and correlative modifications are plainly adaptations to the nature of the surroundings in all branches of the animal kingdom, shows this to be sound reasoning. Among some of these types there are all kinds of metamorphoses and very complicated modes of development, so that it is not difficult to surpass even those of insects. One can apply a similar nomenclature and the same laws in explanation of the often curious and sometimes extraordinary metamorphoses, and these changes are often, as in Taenia, accompanied by corresponding acceleration and loss of primitive stages. The curious transformations of Echinodermata are plainly adaptations of the larvae to a free life in the water before they become attached or sink to the bottom and begin their proper life as crawlers. In this class there are a number of examples of acceleration (Comatula, Spatangoids, etc.). Such life-histories and those of Epicauta, Sitaris and Meloe among beetles which run out the gamut of changes from the simplest Thysanuriform larva through several grub stages to the quiescent pupa, show that the most complicated metamorphoses, called hypermetamorphoses by entomologists, must have arisen in response to the changes of the surroundings. No other hypothesis can account for the number, variety, and novelty of these metamor- 
phoses and their suitability to the number, variety, and novelty of the changes in the surroundings and the corresponding changes in habits of the larvae at different stages of growth.

The occupation of the larval stages by strange and curious forms, like caterpillars, grubs, etc., naturally attracts attention and at first makes one wonder at the apparent eccentricities of nature's ways. But in reality they serve to throw a strong side light upon the normal mode of action of the laws of heredity, and show us that, in spite of its enormous conservative force, heredity is subservient to the effects of habit or use of parts.

That these secondary larval forms are more reduced, although more specialized organisms than the primitive Thysanuriform larvae, has already been stated. Among Coleoptera and Neuroptera this is obvious whenever the Thysanuriform and secondary adaptive forms are present in the growth of the same individual. No one can compare the swollen, soft, round-bodied grubs with the active Thysanuriform larva, especially when occurring in the growth of the same beetle, without realizing that the former is due to specialization by reduction. That their structures, although degraded by this process, are suitable to the conditions under which they live has been pointed out by many writers; notably, Graber, Riley, Lubbock, and Packard. This reduction becomes still more apparent when we regard the larvae of Diptera and the grubs of the weevils among Coleoptera, the latter being generally without legs, and the former also deficient in these organs and in large part without a differentiated head. If these or the caterpillars or other secondary larval forms similar to them were isolated, and their subsequent development into pupae and adults unknown, naturalists would no admit that they possessed close affinities with the adult insects of the same groups, and they would be considered as more rudimentary or simpler in structure than any Thysanuran or Thysanuriform larva. In the most specialized forms of Coleoptera, the weevils, the early development of a footless grub, a reduced form similar to the maggot of the Diptera, replaces both the Thysanuriform larva and also the active sixfooted grub of the normal groups of beetles. The Insecta furnish such apparently isolated examples, and, on account of the absence of intermediate forms, it has been supposed that these could be put in evidence against the derivation of the orders of which they were members from Thysanura, as has been stated above with reference to the saltatorial Orthoptera, but the researches of Brauer, Packard, and Lubbock, demonstrating that the secondary larval stages, grubs, maggots, etc., are modifications of the Thysanuriform larval stages, show that this use of them cannot be admitted. If this be granted, it becomes possible to account for the phenomena as follows. The modified, and adaptive, larval characters of the grubs, caterpillars, etc., havmg become fixed in the organization of such groups as 
the weevils among Coleoptera, and in some whole orders, as in the Lepidoptera and Diptera, have been inherited at such early stages in accordance with the law of acceleration in development that they have replaced the useless Thysanuriform stage. In other words, the absence of this primitive larval stage in the young of many specialized forms of insects now living is due to the tendency to earlier inheritance of the later acquired, adaptive characters of the secondary larval forms.

It is very important for these considerations to notice that after the insects possessing the indirect modes of development have passed through their reductive secondary larval stages, they return to the more normal or direct mode of development in the pupa. In doing this, they clearly illustrate the exceptional and adaptive nature of their deviations from the direct mode during the larval stages, and show that this resumption of the older beaten path marked out by heredity is essential in order that a typical hexapod form may be evolved in the adult stage. The pupa is always a six-legged form, with the legs more or less developed, and being common to all insects, whether quiescent or active, is really a part of the direct mode of development wherever it occurs. It is as universal and essential as are the typical ovarian and adult stages. Indirect development is, therefore, composite. It is first a deviation in the larva from the direct mode, and then a return in the pupa of the direct mode, and this return necessarily brings the organism back again into the normal line of evolutionary changes, and the normal form of insect is the result of this return and the resumption of progressive specialization.

The reverse of this process, i. $e$. when direct development is not resumed, is shown in the case of parasites like the female of Stylops.

If it be true that the stages of development in individuals are abbreviated records of the modifications undergone by the group during its evolution in time, and that as a rule the characteristics of adults of the more generalized or primitive forms of any order, or even of smaller divisions, in all groups of the animal kingdom, show a tendency to occur in the young of more specialized forms of the same group or division, it follows, that in each natural group the specialized forms have been evolved from the generalized forms. This ten. dency to accelerate and abbreviate the record preserved by heredity in the growth and development of each individual can be understood if one imagines a series of forms evolving in time. First, the representatives of the simple, primitive ancestor; then one form after another coming into being successively would each introduce some novel modifications, according to its place in time and the structural series. These modifications being inherited at earlier stages in descendants than those in which they originated in the ancestral forms, would crowd upon the characteristics already fixed by heredity in the growth of the young. By and by, as characteristics 
accumulated, it would become not only inconvenient to repeat all the characteristics of its ancestors, but it would be a physical impossibility for any individual to reproduce them all in the same succession in which they had arisen; life would not be long enough nor vital powers strong enough to accomplish such a process. Nature provides for such emergencies by a law of replacement; and as stated above, when a part or characteristic becomes useless, if it stand in the way of the development of other parts or other characteristics of the same part, it is replaced to a greater or less degree by the newer and more useful modifications. This is the rule so far as relates to an ordinary normal series of forms when such a series can be traced with abundant materials through a sufficiently long period of geologic time, as has been repeatedly shown by Cope and one of the authors. Made confident by such experiences we do not hesitate to apply it to the insects where positive evidence of this sort is not yet forthcoming.

If this be correct, it is evident for example that the sucking-tube and other correlative internal modifications originated in the pupal or adult stages of the primitive Hemipteron, then became fixed in the organization of the order, and are now inherited at an early age, having replaced or driven out the ancestral, primitive, perhaps Thysanuriform mouth parts from the larval stage. The assumption that the sucking mouth parts originated in the pupal or adult stages is considered probable, because, al- though there are many exceptions, characteristics usually originate in the later stages in other branches of the animal kingdom. In Lepidoptera and Diptera, which resemble the Hemiptera in having the highly modified mouth parts with a tubular arrangement, these characteristic peculiarities are confined to the later stages of development, and are not found in their larvae. The larvae of Hemiptera are also decidedly Thysanuriform, and that they originated from a modified Thysanuroid form having biting mouth parts in the larvae and sucking mouth parts in the later stages, seems to be indicated by this fact. We have already seen in such examples as the locusts, etc., that an earlier development in the inheritance of the characters of adults may effectually obliterate the Thysanuriform larva, and in the Coleoptera, Neuroptera, etc., that it is the earlier inheritance of the secondary larval characteristic which accomplishes this result. In no case do the pupal or adult characteristics become accelerated in development so as to replace the larval stage in the second series of orders except in parasites such as the parasitic Pupipara (ticks). The young are in some of these species born as pupae, and the ovarian and larval stages are passed within the mother.*

As a rule, then, the orders having in-

*Among the orders having the direct mode of development a similar case to the Pupipara is to be found in the plant-lice. These being viviparous, the young are born in an advanced stage, and are in reality, although wingless, comparable with active pupae. In the case of the sexually perfect forms which emerge from pseudova, they are, according to Comstock, in a still more advanced condition. 
direct modes of development do not show to any marked extent acceleration in the inheritance of adult or adolescent (pupal) characters, but, on the contrary, the characteristics of these later stages remain remarkably constant in the ages at which they are inherited. They do not encroach upon or replace the larval stage to any very marked extent, as in the examples cited above, among the Orthoptera or Hemiptera. This might be considered as fatal to the application of the law of acceleration, and this would be the case if that law were anything more than the expression for a general result of causes which underlie the action of heredity. One of these causes is what we have already expressed as a law of replacement.

Two modifications cannot occupy the same space, and the secondary larval forms having become fixed in the organ. ization, they hold their own in the development of individuals against the encroachment of the pupal and adult characters by virtue of their suitability and the conservative power of heredity. The few cases in which acceleration of the pupal stages at the expense of the larval stages does take place in the second series of orders seem to show this, since they occur not in the normal forms having the ordinary habitat, but in parasites like the Pupipara.

Teachers who read Sir John Lubbock's interesting chapter on the Nature of metamorphoses will find opposite views expressed in regard to the rank of metamorphoses, and these may confuse them unless explained. He speaks, on page $4 \mathrm{r}$, of the maggots of flies as belonging "to a lower grade" of metamorphoses than the grubs which have biting mouth parts and heads, and of the caterpillar as on a higher level than the vermiform larvae of Diptera and Hymenoptera. This, literally translated, means that larvae, like those of the grubs of most Coleoptera and Lepidoptera, have heads, mouth parts, and legs which have not yet suffered from reduction; but in speaking of these as "lower grade," Lubbock makes a mistake in systematic perspective. .If, as he holds, the secondary larvae are all primarily the outcome of the Thysanuran form, they are all what he ought to call "higher grade," being more specialized and farther removed from this primitive insect standard than the larvae of the more generalized or first series of orders. The same and, we think, more philosophical mode of dealing with the facts leads to the corollary that among themselves the larvae of the more specialized orders are really "higher," if the use of this word is considered essential, or more specialized in proportion to the extent of their structural deviation from the Thysanuran standard. Thus the larvae of Diptera are, as a rule, more specialized than any other, and have to be set on the extreme left in our table on this account. 'The words "higher and lower grade" are extremely confusing, since they embrace three different classes of ideas,-anatomical and physiological facts and teleological notions. Nature leads us along lines of modification which sometimes rise 
through continuous progressive specialization to more and more differentiated structure with correspondingly increased functional powers, or larger or different fields of work. At other times it may lead us in a wave line, which follows a devious course, rising part of the time through progressive specialization, and then falling for another period of time through specialization by reduction. If the animals under consideration be parasites, they may continue on this descending plane both in the growth of the individual and the evolution of the group. Nevertheless the resulting adult is not necessarily of "low grade" in any scientific scheme of arrangement founded upon the principles of evolu- tion. It is, however, farther removed from the primitive type, and is extremely specialized. The use of the aesthetic terms "low" and "high" have come from a period in the history of our science when nature was made to assume a rigidly progressive aspect, each division of the animal kingdom representing a finger-post pointing towards the socalled perfect animal, man, each rising higher and higher in the scale of perfection whose standard was the human organization. Such artificial ideas revenge themselves, and words become their ready instruments, first to express what is false, and then to help in binding the mind with the conservative fetters of habit.

ON THE LIFE HISTORY OF DIABROTICA I2-PUNCTATA, OLIV.

BY H. GARMAN, LEXINGTON, KY.

\section{(Concluded from p. 3o.)}

THE INJURY TO CORN.

The larva of this insect works much like its congener, $D$. longicornis, commonly destroying the roots, but often also working on the underground part of the stalk. The larva of $D$. long $i$ cornis often makes a longitudinal burrow in a root leaving little outward trace of its presence. The larval D. I2-punctata has not been observed to work in this manner, the roots being mined and channeled irregularly, often bored through from side to side, or even completely devoured where the worms are abundant. Very few fields in Kentucky are entirely free from injury, and many are affected very injuriously, the damage being perceptible to casual observation in the retarded growth, and, as the seasons advance, in the prostration of infested corn by the winds. As a rule the injury is greatest on land that retains moisture longest. On high and welldrained land it is not so prevalent. In 

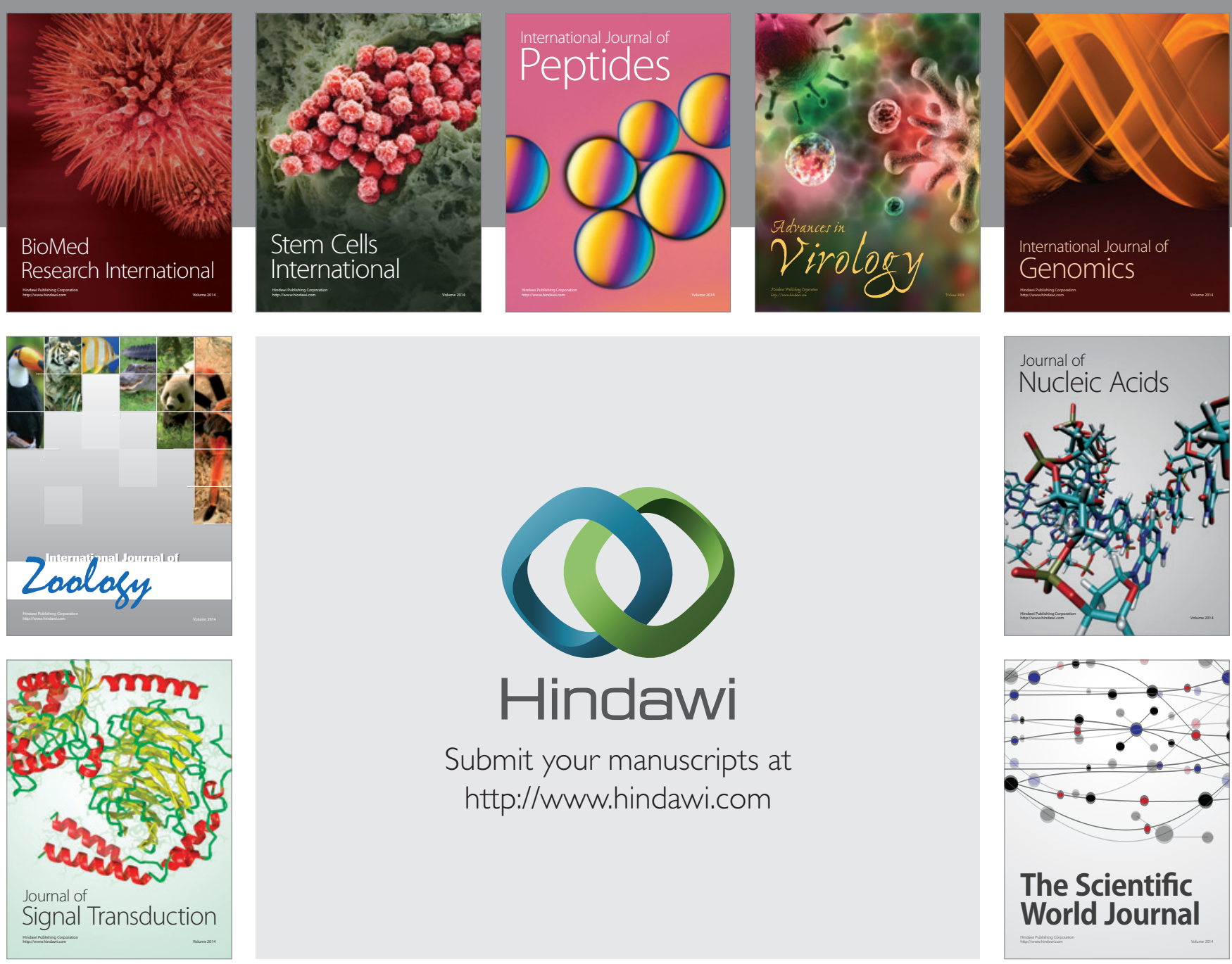

Submit your manuscripts at

http://www.hindawi.com
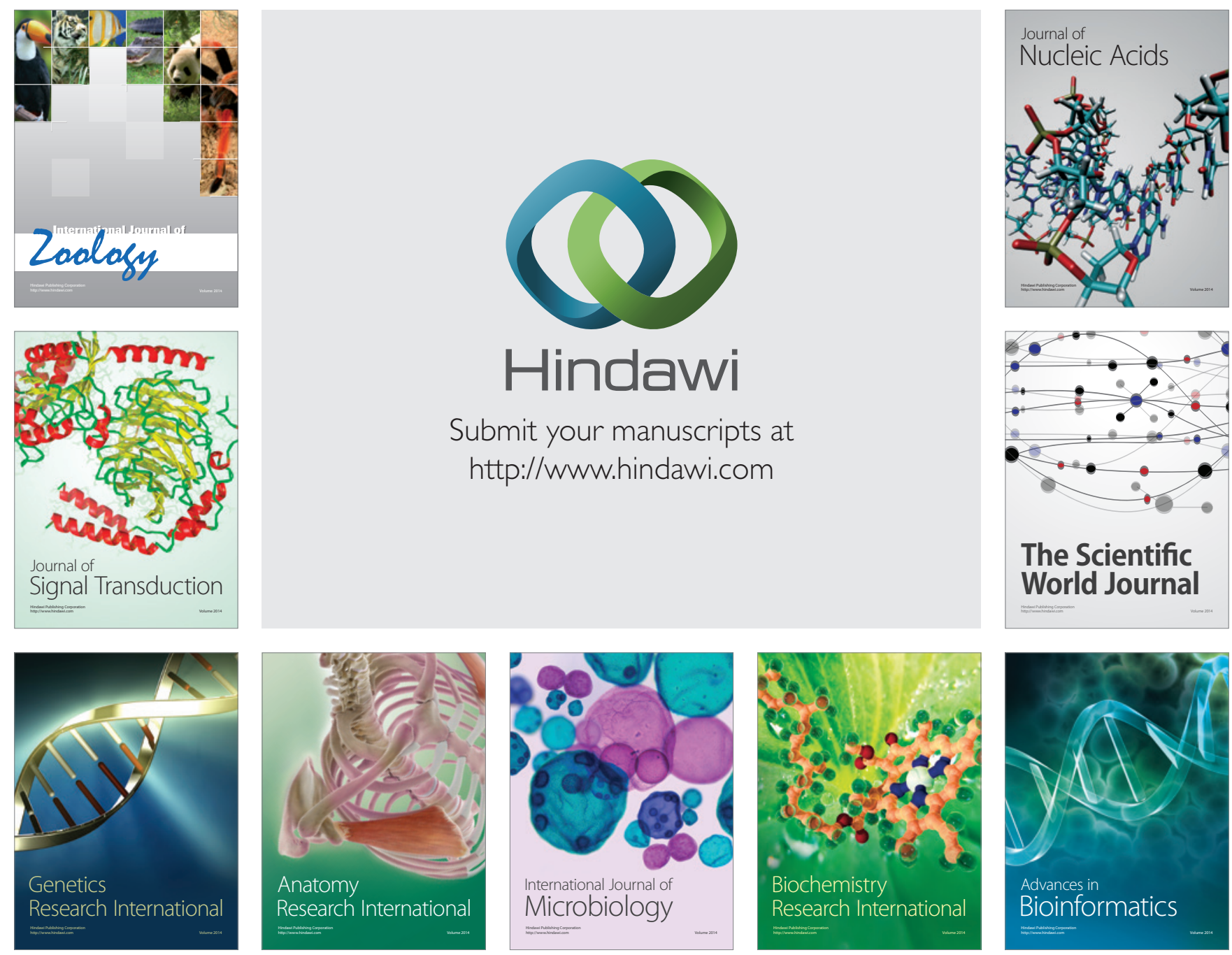

The Scientific World Journal
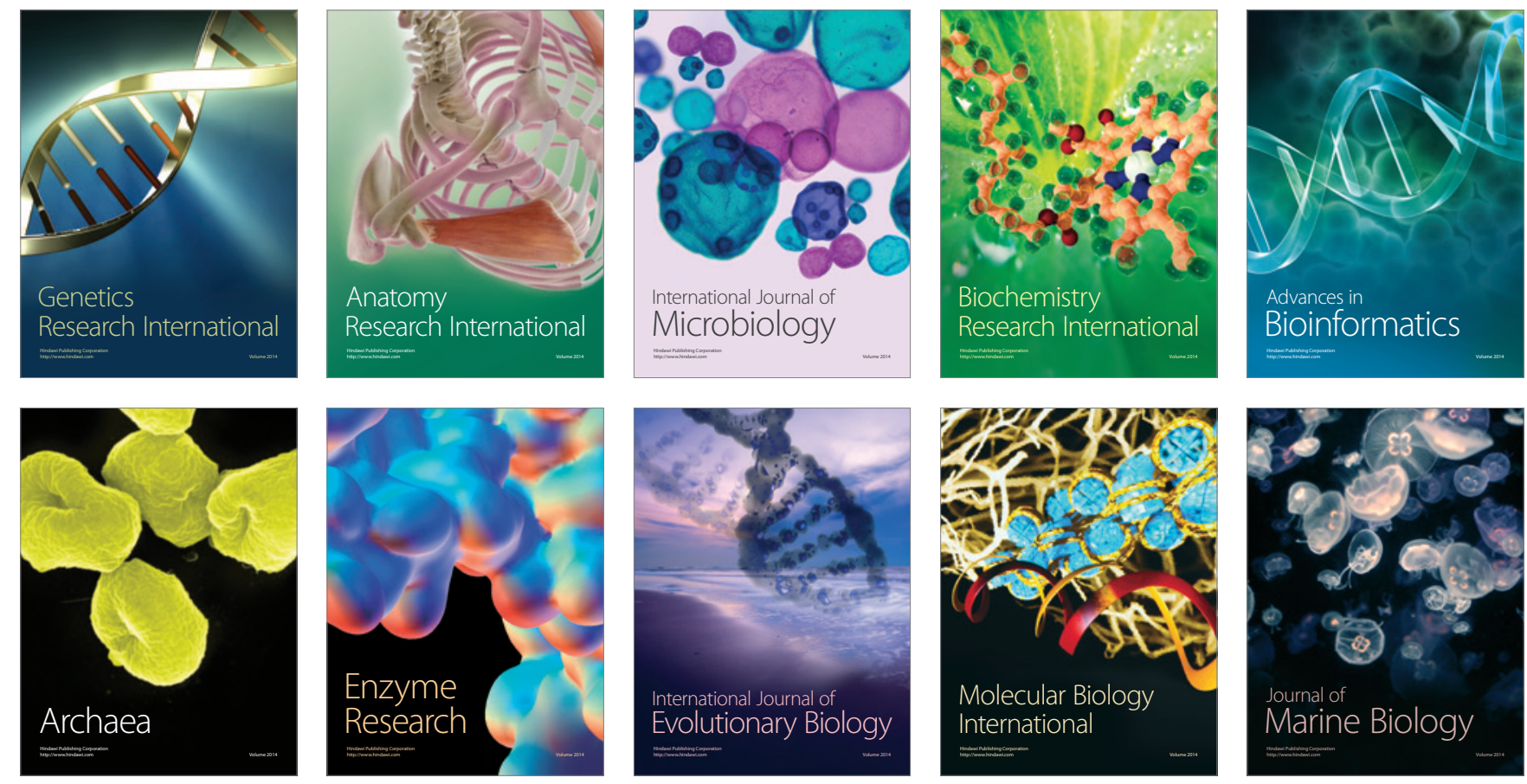